DOI: $10.3901 / J M E .2019 .10 .122$

\title{
基于软篎分停止准则的改进经验模态分解 及其在旋转机械故障诊断中的应用"
}

\author{
彭丹丹 ${ }^{1}$ 刘志亮 ${ }^{1,2}$ 靳亚强 ${ }^{1}$ 秦 勇 $^{2}$ \\ (1. 电子科技大学机械与电气工程学院 成都 611731;
}

2. 北京交通大学轨道交通控制与安全国家重点实验室 北京 100044)

\begin{abstract}
摘要: 笁分停止准则是影响经验模态分解在高速列车旋转机械故障诊断准确率的关键因素之一。目前普遍采用预先设定阈值 的方法, 不具有自适应性, 导致经验模态分解易出现模态混叠的问题, 进而影响故障诊断结果。鉴于充分论证笁分停止准则 对经验模态分解结果的影响, 提出一种能够自适应控制篎分过程的软笚分停止准则, 用于抑制模态混叠问题, 提高经验模态 分解精度和效率。针对目标信号, 该准则通过定义一个刻画全局能量和局部冲击的目标函数, 结合启发式搜索机制, 实现每 次篎分过程中篮分迭代次数的优化, 进而保障经验模态分解获取最优分解结果。利用仿真数据和凯斯西储大学轴承基准数据 集, 对改进的经验模态分解与两种传统实现方法在不同的分解和诊断性能维度上进行对比讨论。最后, 将提出的改进经验模 态分解方法成功应用于高速列车旋转机械模拟试验台的故障诊断案例中。
\end{abstract}

关键词: 经验模态分解; 模态混叠; 笁分停止准则; 故障诊断; 高速列车

中图分类号: TG156

\section{Improved EMD with a Soft Sifting Stopping Criterion and Its Application to Fault Diagnosis of Rotating Machinery}

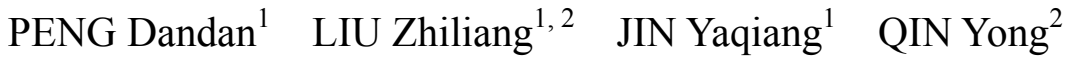

(1. School of Mechanical and Electrical Engineering,

University of Electronic Science and Technology of China, Chengdu 611731;

2. State Key Laboratory of Rail Traffic Control and Safety, Beijing Jiaotong University, Beijing 100044)

\begin{abstract}
The sifting stopping criterion of empirical mode decomposition is one of the key factors affecting the accuracy of fault diagnosis of rotating machinery in high-speed trains. The current method of determining thresholds in advance is generally adopted, which is not adaptive. Such methods lead to the problem of mode mixing in empirical mode decomposition, which affects the fault diagnosis results. The influence of sifting stopping criterion on the empirical mode decomposition results are fully demonstrated, and then a soft sifting stopping criterion that can adaptively monitor the sifting process is proposed. This criterion is used to suppress the mode mixing problem and improve the accuracy and efficiency of empirical mode decomposition. Aiming at the target signal, the criterion defines an objective function that describes the global energy and local impact characteristics, and combines a heuristic mechanism to realize the optimization of the sifting iterations number in each sifting process, so as to guarantee the empirical mode decomposition to obtain the optimal decomposition results. Based on the simulation data and the Case Western Reserve University Bearing Data, the improved empirical mode decomposition is compared with the two traditional methods in different decomposition and diagnostic performance dimensions. Finally, the proposed improved EMD is successfully applied to the fault diagnosis case of rotating machine simulation test rig of high-speed trains.
\end{abstract}

Key words: EMD; mode mixing; sifting stopping criterion; fault diagnosis; high-speed train

* 国家重点研发计划(2016YFB1200401-106)和国家自然科学基金 (51505066，61833002)资助项目。20180529 收到初稿, 20190218 收到修 改稿 


\section{0 前言}

随着高速列车运营速度的不断提高, 人们对高 速列车安全性能的要求也随之提高。旋转机械作为 列车的关键部件, 其运行状态对列车的行车安全性 有至关重要的影响。如果旋转机械出现故障, 轻则 出现意外停车, 重则引发安全事故, 因此对高速列 车旋转机械进行故障诊断十分重要且必要。

故障诊断的关键是从状态监测信号中提取故障 特征。轨道车辆运行环境恶劣、工况复杂, 故障模 式多样 ${ }^{1]}$, 因此故障振动信号复杂, 频率成分丰富, 干扰成分多, 具有非线性和非平稳性等特点。时频 法是处理非线性、非平稳信号的有效方法, 典型的 时频法有维格纳-威尔分布(Wigner-ville distribution, WVD)、短时傅里叶变换(Short-time Fourier transform, STFT)和小波变换等。然而, 这些方法都具有自身 的局限性。其中, WVD 在处理多分量信号时存在 严重的交叉项缺陷 ${ }^{[2]}$; STFT 的时间分辨率和频率分 辨率不能同时兼顾 ${ }^{[3]}$; 小波变换不具有自适应能力, 而且频域部分边缘处出现能量泄露和频率分辨率相 对粗粮等问题 ${ }^{[4]}$ 。经验模态分解(Empirical mode decomposition, EMD) 是由 HUANG 等 ${ }^{[5]}$ 于 1998 年 提出的一种自适应的处理非线性、非平稳信号的时 频分析方法, 它将一个复杂的非平稳信号自适应地 分解为有限个本征模态函数 (Intrinsic mode function, IMF)和一个趋势项残差分量之和, 因此 EMD 方法在高速列车旋转机械故障诊断方面得到 了广泛应用。ZHAO 等 ${ }^{[6]}$ 使用 EMD 方法提取高速列 车走行部抗蛇行减振器和齿轮箱的振动信号故障特 征。为抑制 EMD 的模态混叠问题, 何晓琴等 ${ }^{[7]}$ 采集 模拟高速列车工况试验台上的齿轮不同故障的振动 信号, 使用集合经验模态分解(Ensemble empirical mode decomposition, EEMD)技术, 提取故障信号特 征。进一步, 秦娜等 ${ }^{[8]}$ 为了检测列车转向架减振器 的工作状态, 将 EEMD 和样本熵特征相结合提取振 动信号故障特征。而翟冰等 ${ }^{[9]}$ 将 EEMD 与能量矩特 征相结合, 进行故障特征提取。在此基础上, 宋神 ${ }^{[10]}$ 提出了完备集合经验模态分解 (Complete ensemble empirical mode decomposition, CEEMD)方法, 不仅 抑制了模态混叠问题，而且完备性和自适应性更好。 井波 ${ }^{[11]}$ 则对横向减振器和抗蛇行减振器性能退化、 轨道弯曲不平顺和横向减振器不同失效个数等问题 进行了研究, 比较得出基于 EEMD 排列熵的方法优 于基于 EEMD 关联维数的方法。

虽然在高速列车故障诊断方面, EMD 及其改进
的 EMD 方法得到了广泛的应用, 但在理论上, EMD 仍存在欠包络和过包络、端点效应和模态混叠等问 题。因此在提高 EMD 的分解精度和效率上, 很多 学者致力于研究这三方面参数, 分别是: 包络曲线 拟合、边界条件和篎分停止准则。

对于包络曲线拟合参数, EMD 方法采用三次样 条函数构造包络曲线，存在欠包络和过包络问题。 欠包络指包络曲线进入到了信号内部, 没有完全把 信号包络进去; 过包络指包络曲线的值明显大于对 应信号的极大值或明显小于对应信号的极小值。包 络曲线选取恰当与否直接决定了原始数据能否被很 好的拟合, 这对 EMD 的分解结果有直接的影响, 改善包络曲线对于解决 EMD 模态混叠问题有很大 帮助。因此, 很多学者寻求更优的包络曲线解决欠 包络和过包络问题，如 B 样条算法 ${ }^{[12] 、}$ 、分段三次多 项式贝塞尔插值算法 ${ }^{[13]}$ 、高次样条插值法 ${ }^{[14]}$ 和最小 长度约束法 ${ }^{[15]}$ 等。对于边界条件参数, 该参数直接 影响篎分过程中每次迭代过程的包络曲线拟合。边 界处理不当甚至会引起 EMD 的端点效应问题。鉴 于此, 目前大多数学者在边界条件方面做了相关研 究, 提出了一些方法。例如: 特征波法(Characteristic waves $)^{[5]}$ 、镜像延拓法 ${ }^{[16]}$ 、神经网络法 ${ }^{[17]}$ 、比边界 延拓法(Ratio boundary extension) ${ }^{[18]}$ 和端点优化对称 延拓法(End optimization symmetric extension) ${ }^{[19]}$ 等。

对于篮分停止准则参数, 该参数对每次篮分过 程的篎分迭代次数有直接影响。如果篮分迭代次数 太少, 即 “欠篮”，可能导致一个 IMF 中存在多个 单分量信号; 如果篮分迭代次数过多, 即 “过篮”, 可能导致一个单分量信号位于多个 IMF 中。以上两 种情况即 EMD 模态混叠问题的表现形式, 因此, 研究篎分停止准则参数对于抑制 EMD 模态混叠问 题至关重要。

从上文中发现, 对前两方面参数的研究很多, 但对篮分停止准则参数的研究较少。尽管 HUANG 等 ${ }^{[5]}$ 在 1998 年提出的 EMD 方法中阐述了篮分停止 准则, 然而该准则是一种理想情况, 很难在计算机 数值计算中实现。近年来, 一些学者对篮分停止准则 参数做了一些研究, 如标准差准则 ${ }^{[5]} 、 3$ 阈值准则 ${ }^{[20]}$ 和正交判别准则 ${ }^{[21]}$ 等。这些方法简单直接, 均使用 一个目标函数确定篎分过程的迭代次数, 因此需要 人为预先设定阈值。在本文中, 将这种人为预先设 定阈值的方法定义为硬篎分停止准则, 它具有一定 的局限性, 即需要有先验知识的经验丰富的 EMD 方面专家预先设定阈值。这种硬筛分方法对于实际 的振动信号不具有自适应性, 不同的专家根据自己 经验设定的阈值不同, 因此受人为因素影响很大。 
基于以上所述, 本文借鉴电子科技大学 LIU 等 ${ }^{[22]}$ 在局部均值分解方法中提出的软篮分停止准则的思 想, 对其进行改进并应用到 EMD 中。在此基础上, 对于每次篮分过程, 本文提出的软篮分停止准则能 够自适应地寻找一个最优(或次优)的篮分迭代次 数。该方法定义了一个目标函数, 该函数包括目标 包络信号的全局能量特性和局部冲击特性两个特 征, 分别用方均根和超峭度描述。为了优化该目标 函数, 提出了一个启发式机制, 自适应地确定最佳 筛分迭代次数。最后, 使用仿真数据和凯斯西储大 学轴承基准数据验证了本文提出的软缆分停止准 则在抑制模态混叠, 提高分解精度和效率方面的有 效性, 并将该方法成功应用于轴承和齿轮的故障诊 断中。

本文的组织结构如下。首先, 第 1 节介绍篮分 停止准则参数对 EMD 分解结果的影响。第 2 节就 篮分停止准则参数提出一种软篮分停止准则。然后, 第 3 节应用仿真数据和凯斯西储大学轴承基准数据 验证本文方法的有效性。最后, 第 4 节将本文方法 成功应用于高速列车旋转机械模拟试验台的故障诊 断中。

\section{1 篮分停止准则参数的影响}

EMD 的分解精度和效率依赖于三方面参数: 包 络曲线拟合、边界条件和篮分停止准则。只有准确 合理地选取这三方面参数, 才能抑制或避免 EMD 的欠包络和过包络、端点效应或模态混叠问题。篎 分停止准则参数决定每次篮分过程的篮分迭代次 数, 直接影响 IMF 分量的准确性。如果参数选取不 准确, 可能导致 IMF 分量不准确, 甚至会导致模态 混叠问题。鉴于此, 本文提出一种自适应地确定篎 分迭代次数的软筛分停止准则, 该准则能够有效抑 制 EMD 的模态混叠问题。

为了直观地描述篎分停止准则参数对 EMD 分 解结果的影响, 本节通过一个仿真的多分量调频 (Frequency modulation, FM) 信号说明篮分迭代次数 对 EMD 分解结果的影响, 该信号表达式为

$$
\begin{aligned}
& x(t)=\cos (2 \pi t \times 600+\cos (2 \pi t \times 20))+1.8 \times \\
& \cos (2 \pi t \times 220+1.8 \times \cos (2 \pi t \times 20))+n_{g}(t)
\end{aligned}
$$

式中, $n_{g}(t)$ 是高斯白噪声, 信噪比 (Signal-to-noise ratio, $\mathrm{SNR})$ 为 $20 \mathrm{~dB}$ 。

仿真信号包含两个固有的 FM 单分量信号, 理 论上, 期望通过 EMD 把式(1)仿真信号分解成两个 IMF 分量, 而且和固有的 FM 分量信号尽可能相似。
也就是说, 经过 EMD 分解后, IMF1 只包含频率成 分 $580 \mathrm{~Hz} 、 600 \mathrm{~Hz}$ 和 $620 \mathrm{~Hz}, \mathrm{IMF} 2$ 只包含频率成 分 $200 \mathrm{~Hz} 、 220 \mathrm{~Hz}$ 和 $240 \mathrm{~Hz}$ 。但事实上, 由于噪 声影响, 可能会分解出一些不相关的无意义的 IMF 分量。

EMD 的分解结果易受篎分过程中篮分迭代次 数的影响。如果篮分迭代次数太少，则可能导致一 个 IMF 分量中存在多个单分量信号, 即 “欠篎” 现 象。仿真信号通过 EMD 分解时, 如果每次只篮分 迭代 1 次, 其分解结果出现部分失真, 如图 1 所示。 从 IMF1 和 IMF2 的傅里叶频谱中明显看到: IMF1 包含频率成分 $200 \mathrm{~Hz} 、 220 \mathrm{~Hz} 、 240 \mathrm{~Hz} 、 580 \mathrm{~Hz}$ 、 $600 \mathrm{~Hz}$ 和 $620 \mathrm{~Hz}, \mathrm{IMF} 2$ 包含频率成分 $200 \mathrm{~Hz} 、 220$ $\mathrm{Hz}$ 和 $240 \mathrm{~Hz}$, 其中 IMF2 的频率成分部分能量出现 在 IMF1 中。因此, IMF1 包含两个单分量信号, 出 现了模态混叠问题。

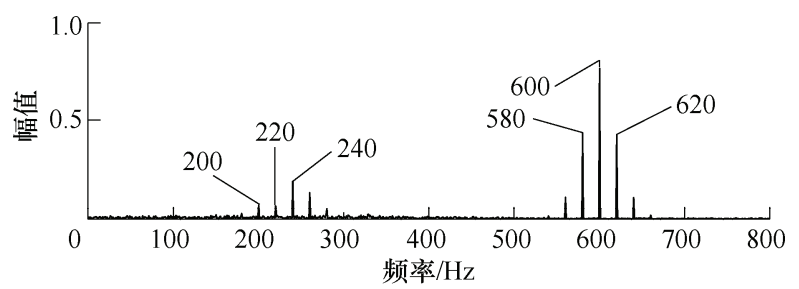

(a) IMF 1

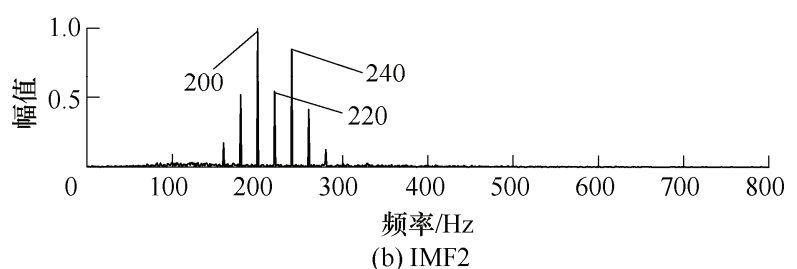

图 1 EMD “欠篮” 分解现象

如果篮分迭代次数太多，则可能导致一个单分 量信号被分解到多个 IMF 中，即 “过篮” 现象。仿 真信号通过 EMD 分解时, 如果每次篮分迭代 200 次, 则 $x(t)$ 被分解为三个 IMF 分量, 但理论上只有 两个固有的 FM 单分量信号, 如图 2 所示。从 IMF2 和 IMF3 的傅里叶频谱中明显看到: IMF2 包含频率

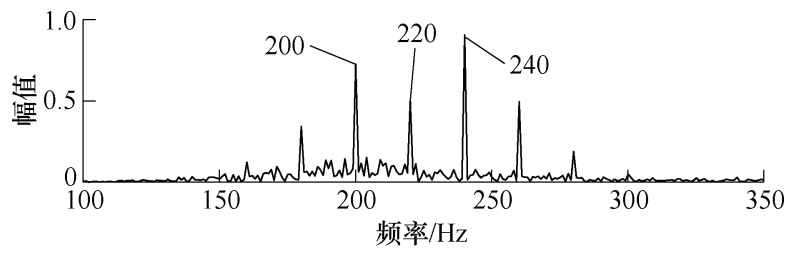

(a) IMF2

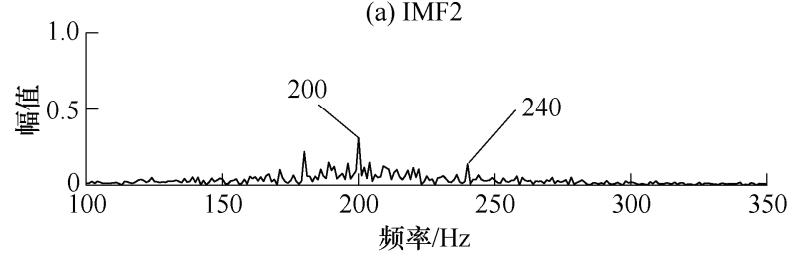

(b) IMF3

图 2 EMD “过篮” 分解现象 
成分 $200 \mathrm{~Hz} 、 220 \mathrm{~Hz}$ 和 $240 \mathrm{~Hz}, \quad$ IMF3 也包含这三 个频率成分, 即第二个 FM 单分量被分解到 IMF2 和 IMF3 中。因此, 一个 FM 单分量信号被分解到 多个 IMF 中, 出现了模态混叠现象。

从上述分析可知, 篮分迭代次数对 EMD 的分 解结果有很大影响, 篮分迭代次数太少或太多都导 致 EMD 的分解结果不理想, 选择能够自适应地确 定最优(或次优)篎分迭代次数的篎分停止准则参数 对提高 EMD 的分解精度具有重要影响。因此, 本 文将进一步提出一种软篮分停止准则。

\section{2 软篣分停止准则}

由第 1 节的讨论可知, EMD 分解结果易受篮分 停止准则参数的影响, 本节提出一种软篮分停止准 则, 能够自适应地确定每次篮分过程的篮分迭代次 数。具体介绍如下。

基于文献[5], EMD 篮分停止准则参数的原始 定义介绍如下。

(1) 极值点的个数和零点的个数必须相等或相 差最多不能超过一个。

(2) 在任何一点, 由局部极大值点形成的包络 线和由局部极小值点形成的包络线的平均值为零。

其中, 条件(2)可以通过式(2)表达, 意味着 $h_{i k}[n]$ 的包络均值信号 $m_{i k}[n]$ 一致地趋向于零

$$
\lim _{k \rightarrow \infty} m_{i k}[n]=0
$$

式中, $n=1,2, \cdots, N_{s}, N_{s}$ 是总的采样点数, $h_{i k}[n]$ 表示第 $i$ 个 IMF 篮分 $k$ 次后的信号, $m_{i k}[n]$ 是 $h_{i k}[n]$ 的包络均值信号, 其中下标 $i$ 表示第 $i$ 个 IMF, $k$ 表 示第 $k$ 次篎分过程。

根据筛分停止准则原始定义中的条件(2), 本文提 出的软笁分停止准则的主要思想包括两方面: (1) 根据 式(2), 应该确定用一个什么样的目标函数描述目标 信号; (2) 如何实现篮分停止准则的自适应机制。

第一方面思路介绍如下。本节从整体能量特性 和局部冲击特性两个角度描述包络均值信号。从整 体能量特性角度, 采用方均根(Root mean square, RMS)评估包络均值信号的整体能量特性。RMS 定 义为: 将一组统计数据平方求和, 求其均值, 再开 平方, 如式(4)所示。如果包络均值信号 $m_{i k}[n]$ 趋向 于零, 则 RMS 值也趋向于零。基于式(2)的原理, 期望 RMS 值尽可能小。从局部冲击特性角度, 采 用超峭度(Excess kurtosis, EK)描述信号可能出现的 局部冲击现象。超峭度是对传统意义上峭度的一个 改进, 将峰度值做减 3 处理, 使得正态分布的峰度
为 0 , 如式(5)所示。如果包络均值信号 $m_{i k}[n]$ 趋向于 零, 则 $\mathrm{EK}$ 值也趋向于零。基于公式(2)的原理, 期 望 EK 值尽可能小。

要评估一个趋向零基准线的包络均值信号, 以 上两个特征是非常重要且必要的。如果只考虑第一 个 RMS 特征, 可能导致包络均值信号出现很多样 本点幅值都很小，而其中几个样本点幅值特别大的 情形, 如图 3a 所示, 其 RMS 和 EK 的值分别是 0.1580 和 997。如果仅考虑第二个 EK 特征, 可能导致包 络均值信号所有样本点都很相似, 但他们的幅值都 远远大于 0 , 如图 $3 b$ 所示, 其 RMS 和 EK 的值分 别是 7.0003 和 1.2354 。这两种情形都降低了篮分 过程的质量, 导致 EMD 的分解结果不准确, 因此 需要同时考虑 RMS 和 EK 这两个特征, 使信号的所 有样本点都一致地趋向于零。

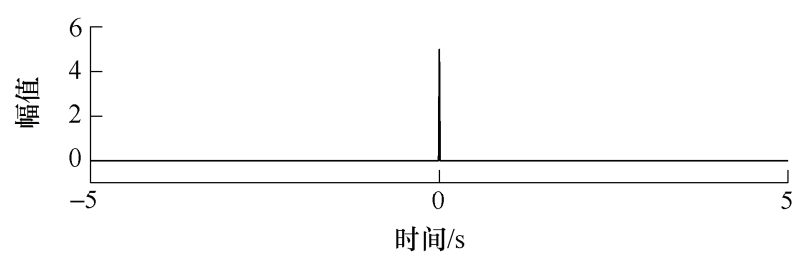

(a) 情形1

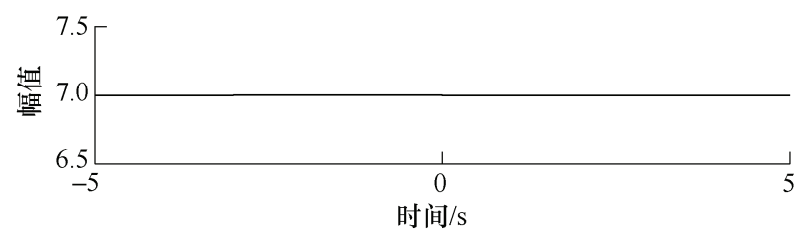

(b) 情形 2

图 3 包络均值信号的两种情形

基于上述讨论，本文提出的描述包络均值信号 的目标函数定义如式(3)

$$
f_{i k}=R M S\left(m_{i k}[n]\right)+\left|E K\left(m_{i k}[n]\right)\right|
$$

式中

$$
\begin{gathered}
R M S_{i k}=\sqrt{\frac{1}{N_{s}} \sum_{n=1}^{N_{s}}\left(m_{i k}[n]\right)^{2}} \\
E K_{i k}=\frac{\frac{1}{N_{s}} \sum_{n=1}^{N_{s}}\left(m_{i k}[n]-\bar{m}\right)^{4}}{\left[\frac{1}{N_{s}} \sum_{n=1}^{N_{s}}\left(m_{i k}[n]-\bar{m}\right)^{2}\right]^{2}}-3
\end{gathered}
$$

式中, $\bar{m}$ 表示 $m_{i k}[n]$ 的算数平均值。

第二方面思路介绍如下。在前言部分总结了近 年来在篮分停止准则方面的研究成果。与这些方法 一样, 可以简单地给式(3)预先设定一个阈值, 以此 建立笁分停止准则, 但这种简单直接的方法需要对 目标包络信号有一个先验知识。如果设定的阈值不 准确, 可能导致 EMD 的分解结果不准确, 也可能 
导致篮分迭代次数太多，运算效率低。

通常, 需要预先设定笁分迭代次数的最大值, 如果篎分迭代次数达到最大值, 强行终止篮分过程, 避免程序出现死循环。然而, 这种篎分终止过程存 在潜在的风险。如果最大值设置太大, 导致篎分迭 代次数过多。一方面很可能引起第二部分所述的“过 筛” 现象, 得到一些与故障无关且没有意义的 IMF 分量, 由此可能导致错误的决策。另一方面增加了 计算时间, 降低了 EMD 的分解效率。通常, 实际 振动信号的信号长度较大, 因此在不影响分解精度 的情况下尽量减小计算时间对于故障诊断效率的提 高具有重要意义。如果最大值设置过小, 导致笁分 迭代次数太少，很可能产生第 1 节所述的 “欠篮” 现象, 对于故障诊断有用的信息可能并未提取出来。

基于上述原因, 根据目标函数式(3), 需要提出 一个启发式机制自适应地确定每次笁分过程的最优 (或次优)篎分迭代次数。提出的启发式机制的笁分 停止准则介绍如下。对于第 $i$ 个 IMF 的某次篮分过 程, $h_{i k}[n]$ 是第 $k-1$ 次平滑后的信号。在每一次迭代 过程中, 目标函数值 $f_{i k}$ 可以通过式(3)计算得到。本 文提出的篎分停止准则是根据连续三次迭代过程的 $f_{i k-2} 、 f_{i k-1}$ 和 $f_{i k}$ 值来决定耖分迭代次数。如果满足两 个条件: (1) 极值点的个数和零点的个数相差不超过 1 ; (2) $f_{i k-2}<f_{i k-1}$ 且 $f_{i k-1}<f_{i k}$, 则篮分过程终止。并且 返回满足上述(1)条件下的第 $k-2$ 次的 $h_{i k-2}[n]$ 作为 $I M F_{i}$ 。否则, 篮分过程一直继续, 直到达到预先设 定的每次篎分过程迭代次数的最大值, 返回最大篎 分次数的 $h_{i k}[n]$ 作为 $I M F_{i}$ 。这种自适应篮分流程如 图 4 所示。其中, $r_{i}[n]$ 表示从信号 $x[n]$ 减去第 $i$ 个 IMF 分量信号 $c_{i}[n]$ 后的残余信号; $m_{i k}[n]$ 表示第 $i$ 个 IMF 的第 $k$ 次篎分过程的上下包络线的均值信号, $P_{1}$ 表 示当 $f_{i k-1}$ 值小于 $f_{i k}$ 值时, 输出为 1 。当判断条件 $P$ 大于等于 2 , 即出现 $f_{i k-2}<f_{i k-1}$ 且 $f_{i k-1}<f_{i k}$ 时, 笁分 停止(图 4)。

\section{3 仿真数据和基准数据验证}

本节通过仿真数据和凯斯西储大学轴承基准数 据对本文方法进行验证, 并且通过把本文方法与 RILLING 方法 ${ }^{[20]}$ 和 WU 方法 ${ }^{[23]}$ 进行对比, 体现出 本文方法在 EMD 分解精度和效率方面的优势。下 面介绍以上三种方法的参数设置: 对于包络估计, 三种方法均采用 HUANG 等 ${ }^{[5]}$ 使用的三次样条曲线 拟合方法; 对于边界条件参数, WU 方法采用 HUANG 等 ${ }^{[5]}$ 使用的特征波法, RILLING 方法和本 文方法采用镜像延拓法 ${ }^{[16]}$, 延拓比设置为 0.2 ; 对

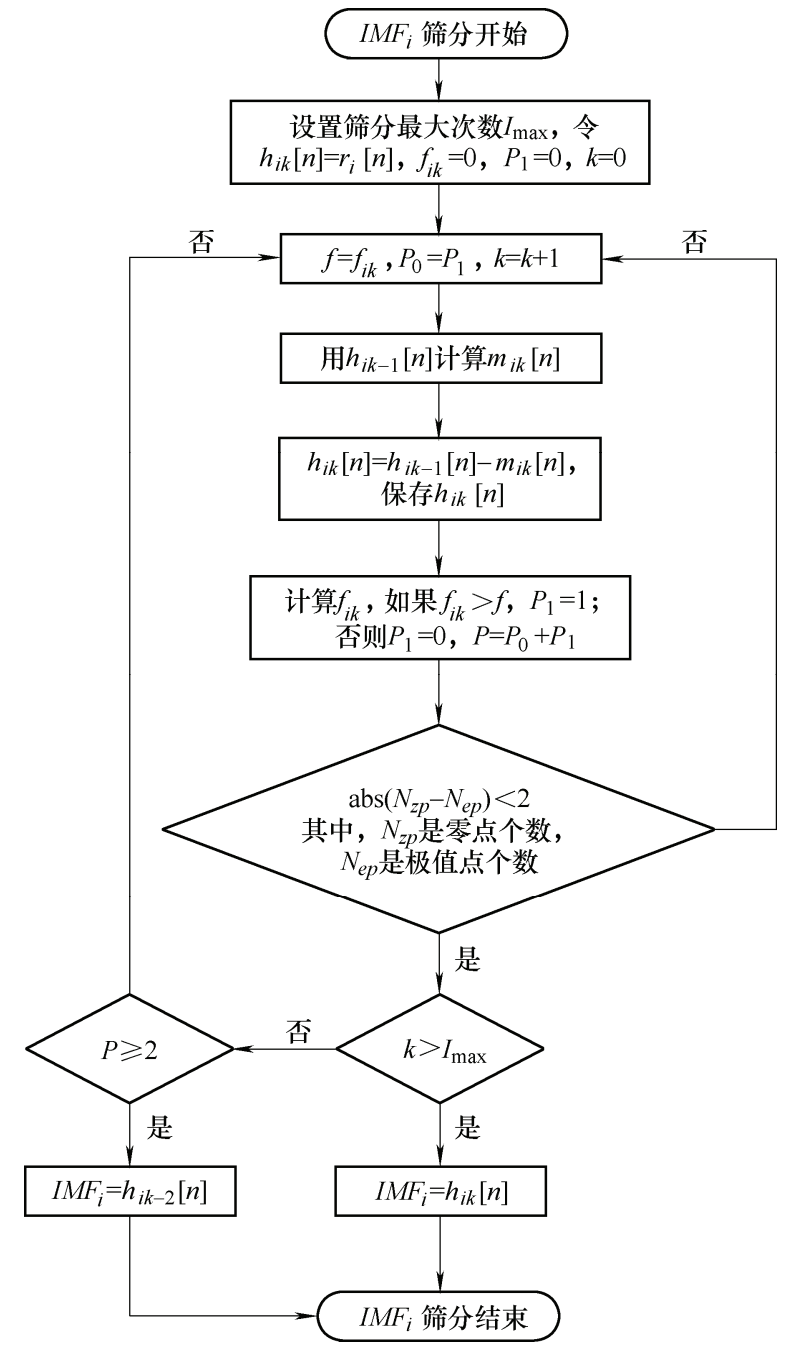

图 4 IMF 篮分过程流程图

于篎分停止准则参数, WU 方法是固定篮分 10 次, RILLING 方法采用 3 阈值准则 ${ }^{[20]}, 3$ 阈值分别设置 为 $0.05 、 0.5$ 和 0.05 , 本文方法采用第 2 节介绍的软 篮分停止准则。其中, RILLING 方法和 WU 方法代 码均采用作者已公开的代码, 本文方法的代码见文 献[24]。

下面通过以下 5 个指标评估三种对比方法的优 劣。5 个指标分别是方均根误差、篮分迭代次数、 IMF 个数、正交性指数和算法时间。其具体内容介 绍如下。

(1) 方均根误差。仿真信号中包含真实的 FM 信 号, 通过方均根误差(Root mean square error, RMSE) 能够评估估计的 FM 信号的准确性, RMSE 值越 小, 表示分解的准确率越高。RMSE 的计算公式定 义如下

$$
R M S E=\sqrt{\frac{1}{N_{s}} \sum_{n=1}^{N_{s}}(x[n]-\hat{x}[n])^{2}}
$$

式中, $x[n]$ 表示原始信号, $\hat{x}[n]$ 表示估计信号。

（2）篮分迭代次数。在某次篮分过程中, 一个 
相对较少的篮分迭代次数隐含以下两点优势: 一方 面提高了 EMD 的分解效率, 另一方面降低了篮分 过程中误差累积的影响, 从而得到更为准确的分解 结果。

(3) IMF 个数。如果 IMF 个数太多, 可能是由 于 EMD 分解得到了一些与故障无关且没有意义的 IMF 分量, 由此可能导致错误的决策, 并且增加了 计算时间, 降低了 EMD 的分解效率。特别在数据 量很大的情况下, 对于算法效率的要求尤为明显。 因此, EMD 分解得到的 IMF 的数量总是期望能和 真实的信号成分数量一样。

（4）正交性指数。虽然 EMD 分解不能保证得到 一个正交的分解结果, 但是正交性指数 (Orthogonality index, OI)仍是一个很重要的指标。 它用来评价分解得到的 IMF 分量是否相互正交, OI 值越小, 表明 IMF 之间的正交性越好。OI 的计算 公式定义如下

$$
O I=\frac{1}{\sum_{n=1}^{N_{s}} x^{2}[n]} \sum_{i=1}^{p} \sum_{j=1}^{k} \sum_{n=1}^{N_{s}} c_{i}[n] \times c_{j}[n]
$$

式中, $c_{i}[n]$ 是 IMF 分量信号。

(5) 算法时间。算法时间反映了 EMD 的分解效 率。算法时间越少, 表明 EMD 的分解效率越高。 本文测试环境中使用了 Intel Core i7-7500 的中央处 理器, $8.00 \mathrm{~GB}$ 的 RAM 存储器和 Windows 10 家庭 版操作系统安装的 Matlab 2015a 运行程序。

\section{1 仿真数据}

本文使用式(1)定义的仿真信号在三种不同的 噪声情况下进行 EMD 分解。这三种噪声情况对应 的 SNR 如下所示: $5 \mathrm{~dB} 、 15 \mathrm{~dB}$ 和 $20 \mathrm{~dB}$, 此处使 用 Matlab 内置的 awgn 函数为仿真信号增加噪声。 仿真信号在三种不同 SNR 情况下, 运行三种方法获 得的五个指标对比结果如表 1 3 所示。总体来说, 本文方法相比于其他两种方法在 EMD 的分解精度 和效率方面占有优势。尤其在 SNR 较小时, 本文方 法分解效果更加显著。

表 1 信噪比为 $20 \mathrm{~dB}$ 下的指标结果

\begin{tabular}{|c|c|c|c|}
\hline 性能指标 & RILLING 方法 ${ }^{[20]}$ & WU 方法 ${ }^{[23]}$ & 本文方法 \\
\hline 成分 1 的 RMSE & 0.2150 & $\underline{0.1929}$ & 0.2352 \\
\hline 成分 1 的笁分迭代次数 & 12 & 10 & $\underline{1}$ \\
\hline 成分 2 的 RMSE & 0.5168 & 0.4431 & $\underline{0.2251}$ \\
\hline 成分 2 的篮分迭代次数 & $\underline{\mathbf{8}}$ & 10 & 30 \\
\hline IMF 个数 & 10 & 11 & $\underline{3}$ \\
\hline OI & 0.1052 & $\underline{0.0363}$ & 0.0752 \\
\hline 算法时间 & 0.3841 & 0.6167 & $\underline{0.1639}$ \\
\hline
\end{tabular}

表 2 信噪比为 $15 \mathrm{~dB}$ 下的指标结果

\begin{tabular}{lccc}
\hline \multicolumn{1}{c}{ 性能指标 } & RILLING 方法 ${ }^{[20]}$ & WU 方法 & 本文方法 \\
\hline 成分 1 的 RMSE & 0.5459 & $\underline{0.4709}$ & 0.5047 \\
成分 1 的篎分迭代次数 & 106 & $\underline{10}$ & 30 \\
成分 2 的 RMSE & 0.6543 & 0.8737 & $\underline{0.6408}$ \\
成分 2 的笁分迭代次数 & 14 & 10 & $\underline{1}$ \\
IMF 个数 & 10 & 11 & $\underline{7}$ \\
OI & 0.0507 & 0.0749 & $\underline{0.0455}$ \\
算法时间 & 0.4637 & 0.6155 & $\underline{0.4022}$ \\
\hline
\end{tabular}

表 3 信噪比为 $\mathbf{5} \mathbf{d B}$ 下的指标结果

\begin{tabular}{lccc}
\hline \multicolumn{1}{c}{ 性能指标 } & RILLING 方法 ${ }^{[20]}$ & WU 方法 & 本文方法 \\
\hline 成分 1 的 RMSE & 0.4475 & 0.4316 & $\underline{0.4267}$ \\
成分 1 的篎分迭代次数 & 36 & 10 & $\underline{5}$ \\
成分 2 的 RMSE & 0.6346 & 0.4360 & $\underline{0.3123}$ \\
成分 2 的篮分迭代次数 & 14 & 10 & $\underline{6}$ \\
IMF 个数 & 10 & 11 & $\underline{3}$ \\
OI & 0.0657 & 0.0405 & $\underline{0.0369}$ \\
算法时间 & 0.3991 & 0.5991 & $\underline{0.3476}$ \\
\hline
\end{tabular}

从分解精度来看, RMSE 值越小, 表明分解精 度越高。OI 值反映了分解结果的正交性, OI 值越 小, 表明正交性越好, 各个分量间的相关性越小, 模态混叠现象抑制地越好, 这从另一个侧面反映分 解精度越高。此外, IMF 个数和原信号真实分量个 数越接近, 则不相关且无意义的信号分量就越少, 这也表明分解的精度越高。从表 $1 \sim 3$ 的 RMSE 值 来看, 本文方法相比其他两个方法, RMSE 的值更 小。尤其是在成分 2 的分解上, 本文方法的效果整 体上更加显著。当然, 试验结果中也出现部分个例, 但这并不影响对本文方法的整体评价。例如, 在 $\mathrm{SNR}=20 \mathrm{~dB}$ 时, 对于 EMD 分解得到的第一个 IMF 分量的 RMSE 值, 其他两种方法略好于本文方法; 在 $\mathrm{SNR}=15 \mathrm{~dB}$ 时, 第一个 IMF 分量的 RMSE 值, WU 方法略好于本文方法。从表 1 3 的 OI 值来看, 除了 $\mathrm{SNR}=20 \mathrm{~dB}$ 的情况, $\mathrm{WU}$ 方法的 OI 值略好 于本文方法, 而对于其他情况, 本文方法的 OI 值 要远好于其他两种方法。从表 1 3 中所示的 IMF 个数来看, 本文方法的 IMF 个数远小于其他两种 方法。这三个指标都说明了本文方法在 EMD 分解 精度方面都优于其他两种方法, 尤其是在强噪声情 况下。

从分解效率来看, 如果篮分迭代次数越少, IMF 个数越小, 则总的计算时间越少。从表 $1 \sim 3$ 的篮分迭代次数来看, 本文方法几乎都远小于其他 两种方法。从 IMF 个数来看, 本文方法获得的 IMF 个数更少。从算法时间上也验证了本文方法在效率 上比其他两种方法更有优势。这三个指标都说明了 
本文提出的方法在 EMD 分解效率方面具有显著的 优势。

随后, 用式(1)中的仿真信号在 $\mathrm{SNR}=5 \mathrm{~dB}$ 时 的情形举例说明本文方法的实现效果。本文方法对 该仿真信号的分解结果如图 5 所示。可以看出, IMF1 和 IMF2 分别是两个估计的 FM 分量信号, IMF3 是 一个无意义的信号分量, 最后是一个残余分量。从 这个分解结果证实了本文方法在强噪声情况下对信 号分解的鲁棒性。

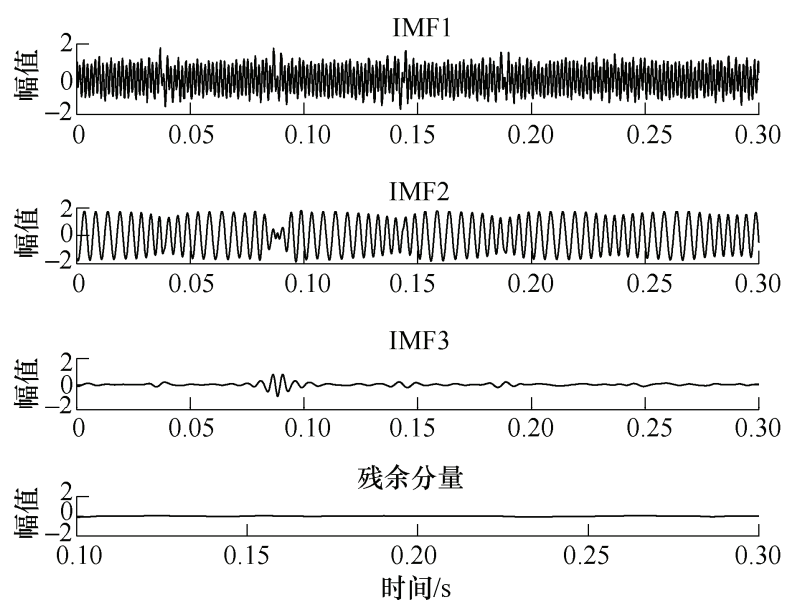

图 5 本文方法获得的 IMF 分量的时域波形图

\section{2 基准数据}

为了进一步验证本文方法的有效性, 本节采用 凯斯西储大学的轴承基准数据 ${ }^{[25]}$ 对本文方法进行 验证。该数据对应驱动端轴承内圈 $0.5334 \mathrm{~mm}$ 裂纹 故障, 在电动机负载为 $0 \mathrm{~kW} 、 0.735 \mathrm{~kW} 、 1.47 \mathrm{~kW}$ 和 $2.205 \mathrm{~kW}$ 四种工况下从驱动端采集的振动加速 度数据。采样频率是 $12 \mathrm{kHz}$, 驱动端轴承内圈的故 障特征频率是 $f_{B P F I}=5.415 \times f_{r}^{0}$, 其中 $f_{r}$ 是转频(本试 验中约为 $30 \mathrm{~Hz}$ )。

用本文方法对四种不同负载下的数据进行 EMD 分解, 并对分解出的每个 IMF 分量进行平方 包络谱分析, 进而选择出对故障敏感的 IMF 分量用 于后续轴承故障诊断, 其平方包络谱见图 6 10。

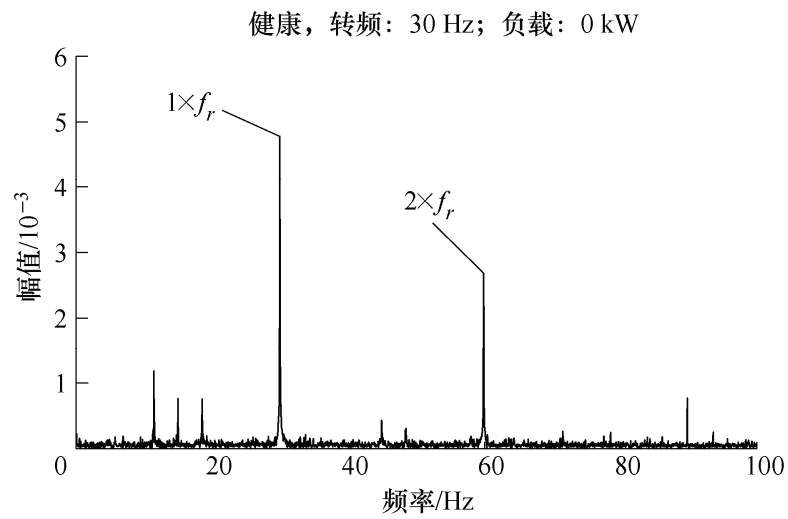

图 6 健康轴承平方包络谱
需要说明的是, 由于自适应选择对故障敏感的 IMF 分量是基于 EMD 的故障诊断方法所面临的另一个 重要问题, 本文中不再展开讨论, 仅采用人工观察 的方式进行选择。图 6 展示了驱动端采集的健康数 据进行的平方包络谱分析, 其中 $1 \times f_{r}$ 和 $2 \times f_{r}$ 清晰可 见。从图 7 10 可以清晰看到, 在四种不同负载工 况下, 平方包络谱在故障特征频率处幅值变化显著, 绝对值远高于健康数据。因此, 可以说明轴承内圈 存在故障，其诊断结果和真实情况相符。

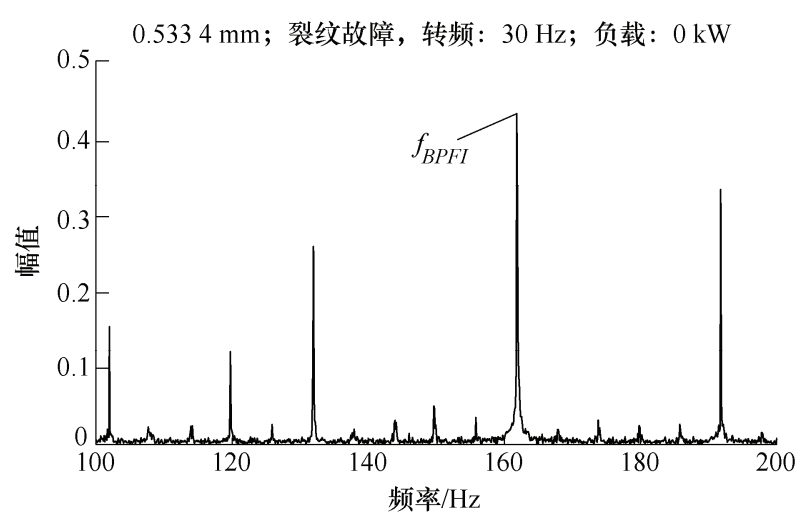

图 7 故障轴承敏感分量平方包络谱

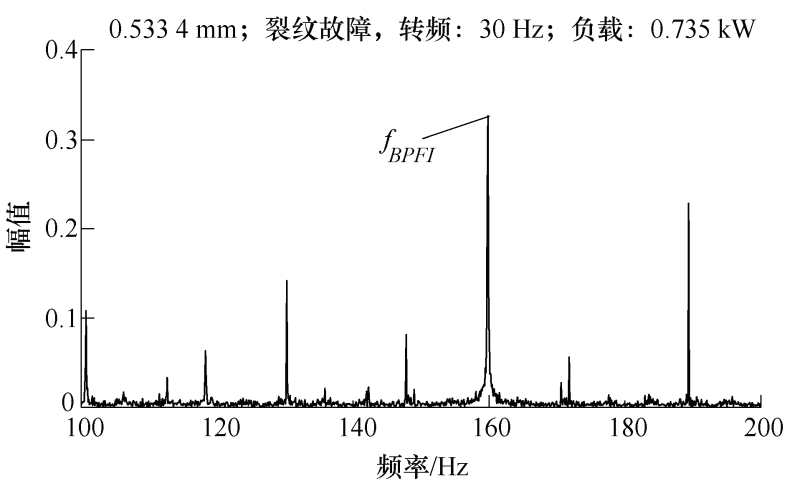

图 8 故障轴承敏感分量平方包络谱

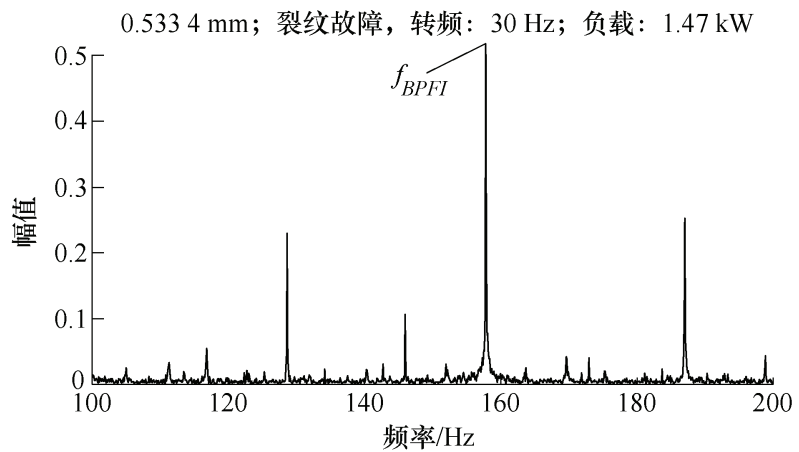

图 9 故障轴承敏感分量平方包络谱

然后分别再用其他两种方法对上述相同的故障 数据进行了 EMD 分解, 并对包含故障特征频率的 IMF 成分进行了平方包络谱分析。通过故障特征频 率处的幅值、IMF 个数、OI 值和算法时间这 4 个指 标对三种方法进行了比较。从表 4 中明显看出, 相 
比其他两种方法, 本文方法在故障特征频率处的幅 值高、OI 值低、IMF 个数少且算法时间少。并通过 选取其他不同的信号进行多次试验发现, 在这 4 个 指标下, 本文方法一般都优于其他两种方法。在保 证精度比其他两种对比方法更高的情况下, 计算时 间有着明显的优势, 运算效率远高于其他两种方法。 因此, 可以预见本文方法在大数据情况下能够发挥 更好的作用。

表 4 不同方法的对比结果

\begin{tabular}{lccc}
\hline \multicolumn{1}{c}{ 性能指标 } & RILLING 方法 ${ }^{[20]}$ & WU 方法 ${ }^{[23]}$ & 本文方法 \\
\hline 故障特征频率 & 0.4320 & 0.4349 & $\underline{0.4375}$ \\
处幅值 & 17 & 15 & $\underline{7}$ \\
IMF 个数 & 0.0103 & 0.0056 & $\underline{\underline{0.0040}}$ \\
OI & 22.9514 & 36.299 & $\underline{11.1963}$ \\
\hline
\end{tabular}

$0.5334 \mathrm{~mm}$; 裂纹故障, 转频： $30 \mathrm{~Hz}$; 负载： $2.205 \mathrm{~kW}$

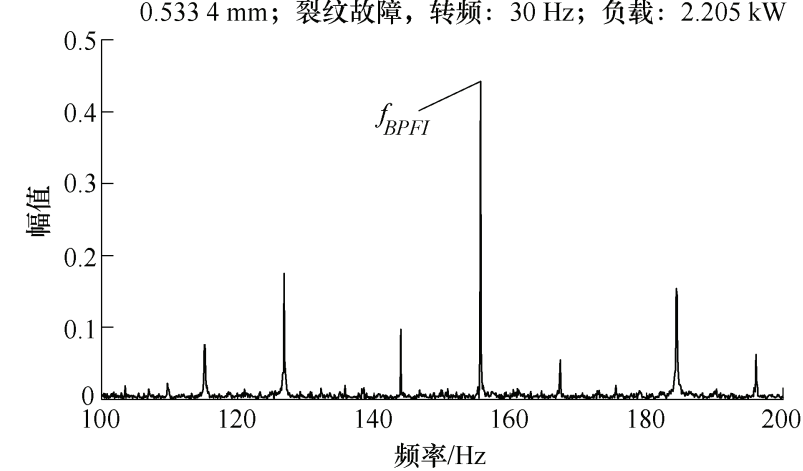

图 10 故障轴承敏感分量平方包络谱

\section{4 高速列车旋转机械故障诊断应用}

高速列车旋转机械的故障诊断对于保障列车的 安全运行非常重要, 因此本节中将本文方法应用于 高速列车旋转机械的故障诊断中。根据旋转机械出 现故障会发生调幅调频现象的共性, 结合高速列车 高速、重载的运行工况, 搭建了一个旋转机械(一级 行星齿轮箱)试验台, 用于模拟高速列车旋转机械的 运行工况。

如图 11 所示, 试验台是由直齿轮箱、一级行星

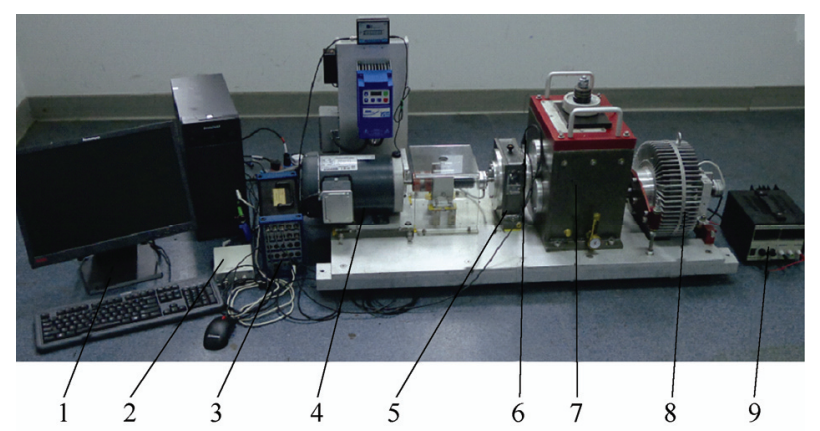

图 11 行星齿轮箱试验台
齿轮箱、 $2.24 \mathrm{~kW}$ 的三相电动机和负载调控装置等 组成。负载通过电磁制动器施加负荷实现, 电磁制 动器的扭矩范围是 $1.355 \sim 88.075 \mathrm{~N} \cdot \mathrm{m}$ 。因为扭矩 与输入电流成正比, 所以本文用电流值代表负载水 平, 不再换算成扭矩值, 本文数据采用的负载水平 为 $1.3 \mathrm{~A}$ 。

行星齿轮箱的物理参数如下: 太阳轮 28 齿, 行星轮 36 齿, 齿圈 100 齿, 行星齿轮的数量为 4 , 传动比 4.75 。行星齿轮箱齿圈固定, 太阳轮是其输入 端。为模拟高速列车旋转机械高速重载的工况环 境, 在重载水平为 $1.3 \mathrm{~A}$ 时, 对带有齿面磨损故障 的齿轮(图 12a)进行了三组不同转频 $(30 \mathrm{~Hz} 、 40 \mathrm{~Hz}$ 和 $50 \mathrm{~Hz}$ )下的故障试验。行星齿轮箱体垂直方向的 加速度传感器用于采集试验过程中产生的振动信 号, 采样频率设置为 $30720 \mathrm{~Hz}$, 采集时间为 $2 \mathrm{~s}$ 。

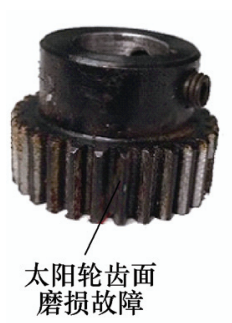

(a)

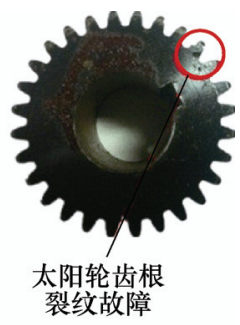

(b)
图 12 太阳轮故障实物图

在本试验中, 太阳轮的故障特征频率是 $f_{\text {sun }}=$ $3.125 \times f_{r}^{0}$ 。因此, 在 $30 \mathrm{~Hz} 、 40 \mathrm{~Hz}$ 和 $50 \mathrm{~Hz}$ 的转频 下, 太阳轮的故障特征频率分别对应为 $93.75 \mathrm{~Hz}$ 、 $125 \mathrm{~Hz}$ 和 $156.25 \mathrm{~Hz}$ 。然后, 用本文方法对采集数 据进行 EMD 分解, 得到若干个 IMF 分量, 对含有 故障特征频率成分的 IMF 分量进行平方包络谱分 析, 如图 13 15 所示。从三个不同转频的平方包络 谱中看到: 在太阳轮故障特征频率及其倍频处的幅 值都出现了明显的增加。因此, 可以推断太阳轮处 存在故障, 此诊断结果和实际情况相符。

齿面磨损故障, 转频: $30 \mathrm{~Hz}$; 负载: $1.3 \mathrm{~A}$

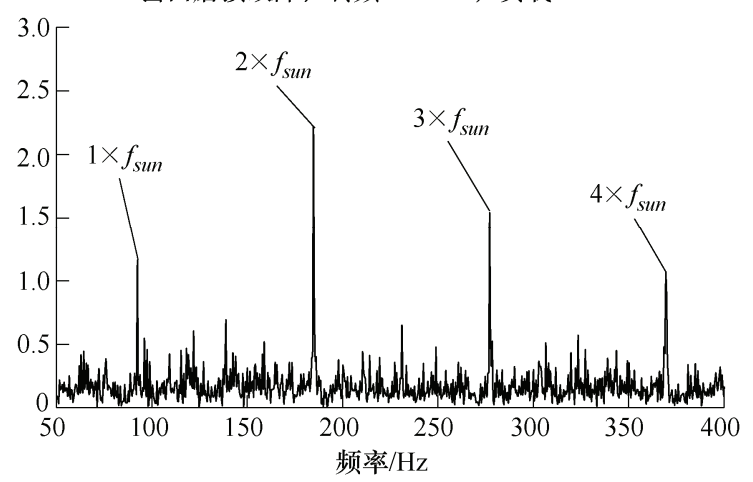

图 13 磨损齿轮敏感分量平方包络谱 
齿面磨损故障, 转频： $40 \mathrm{~Hz}$; 负载： $1.3 \mathrm{~A}$

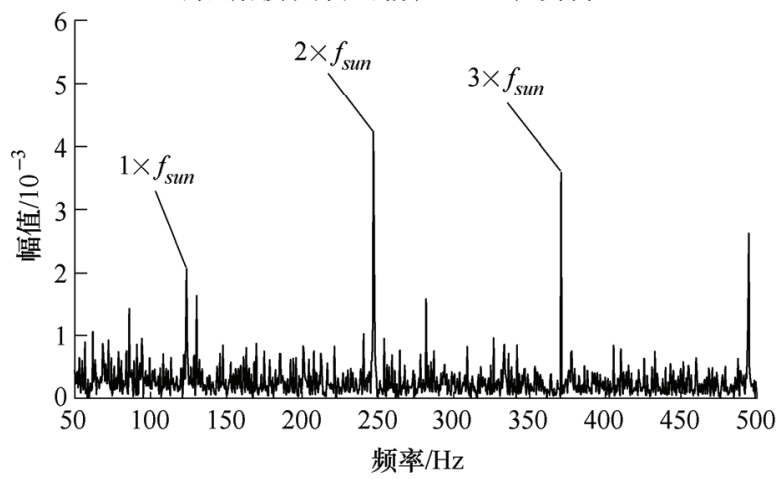

图 14 磨损齿轮敏感分量平方包络谱

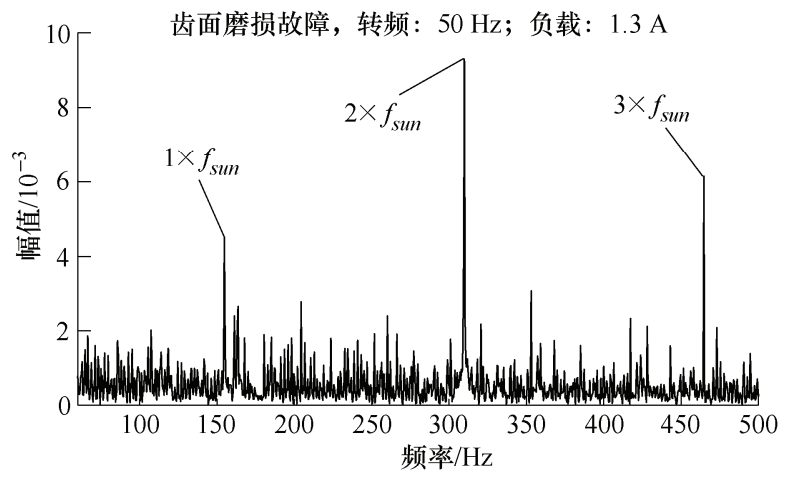

图 15 磨损齿轮敏感分量平方包络谱

除此之外, 其他故障试验也被用于验证本文 方法的有效性, 包括太阳轮齿根裂纹、齿面磨 损和局部断齿等。由于本文篇幅受限, 仅在此 展示了太阳轮齿根裂纹故障(图 12b)的诊断结果。 如图 16 所示, 在转频 $30 \mathrm{~Hz}$ 工况下, 太阳轮故障 特征频率处幅值同样出现了明显增加, 这说明 太阳轮存在故障。综上所述, 本文方法在高速列 车旋转机械模拟试验台上均得出了正确的诊断 结论。

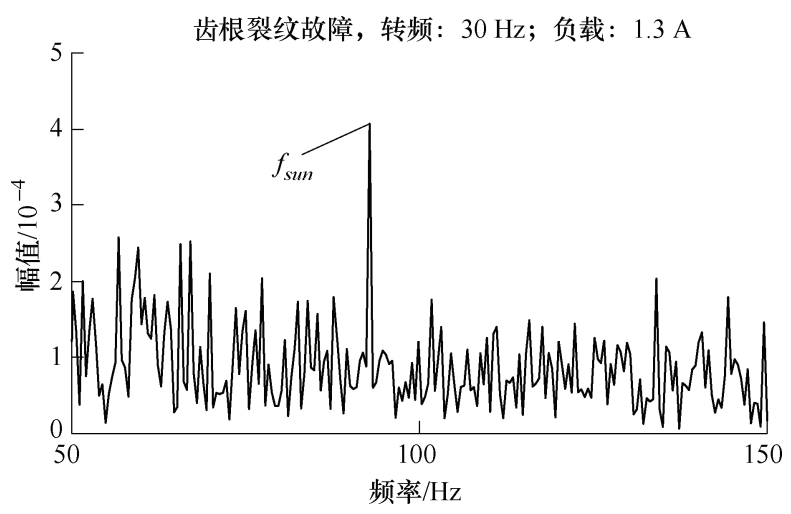

图 16 裂纹齿轮敏感分量平方包络谱

\section{5 结论}

篮分停止准则是影响 EMD 在高速列车旋转 机械故障诊断准确率的关键因素之一。本文以篮 分停止准则为切入点, 对篮分停止准则与 EMD 分 解结果、故障诊断之间的关系进行分析讨论, 并 在 Matlab 平台下实现了该算法, 获得的主要结论 如下。

(1) 探讨了篎分停止准则是影响 EMD 算法的 重要因素之一。它能直接决定笁分次数, 能够直接 影响 EMD 分解效率。不同的笁分次数会导致不同 的分解结果, 因此它又能间接影响分解效果, 与模 态混叠存在因果联系。

(2) 提出的软篮分停止准则能够自适应地决定 篎分过程中的最优篎分次数, 抑制了 EMD 分解过 程中易出现的模态混叠问题，并且该准则不需要先 验知识丰富的专家预先设定, 避免了人为因素对 EMD 分解结果的影响。

(3) 改进的 EMD 算法在仿真数据集上展示出 在分解精度和分解效率方面整体优于两种传统的篎 分停止准则(即 RILLING 提出的 3 阈值准则 ${ }^{[20]}$ 和 WU 提出的固定篎分次数准则 ${ }^{[23]}$ )。同时, 在凯斯西 储大学滚动轴承基准数据集上验证了其在故障诊断 上具有优势。最后, 本文方法成功应用于高速列车 旋转机械模拟试验台的故障诊断案例中。

参 考 文 献

[1] 刘志亮, 潘登, 左明健, 等. 轨道车辆故障诊断研究进 展 $[\mathrm{J}]$. 机械工程学报, 2016, 52(14): 134-146.

LIU Zhiliang, PAN Deng, ZUO Mingjian, et al. A review on fault diagnosis for rail vehicles[J]. Journal of Mechanical Engineering, 2016， 52(14): 134-146.

[2] STASZEWSKI W J, WORDEN K, TOMLINSO G R. Time-frequency analysis in gearbox fault detection using the Wigner-Ville distribution and pattern recognition[J]. Mechanical Systems and Signal Processing, 1997, 11(5): 673-692.

[3] GABOR D. Theory of communication[J]. Journal of the Institution of Electrical Engineers，2011，94(73): 58.

[4] LIN J, ZUO M J. Gearbox fault diagnosis using adaptive wavelet filter[J]. Mechanical Systems and Signal Processing, 2003, 17(6): 1259-1269.

[5] HUANG N E, SHEN Z, LONG S R. The empirical mode decomposition and the Hilbert spectrum for nonlinear and non-stationary time series analysis $[\mathrm{J}]$. Proceedings 
Mathematical Physical and Engineering Sciences, 1998, 454(1971): 903-995.

[6] ZHAO J, YANG Y, LI T, et al. Application of empirical mode decomposition and fuzzy entropy to high-speed rail fault diagnosis[M]. Berlin: Springer Berlin Heidelberg, 2014.

[7] 何晓琴, 常友渠. 一种运用非线性特征提取的动车汼引 齿轮箱故障可靠诊断新方法 [J]. 现代制造工程, 2015 (6): 31-39.

HE Xiaoqin, CHANG Youqu. A novel approach for reliable gearbox fault diagnosis in high-speed train driving system based on nonlinear feature extraction[J]. Modern Manufacturing Engineering, 2015 (6): 31-39.

[8] 秦娜, 金炜东, 黄进, 等. 基于 EEMD 样本熵的高速 列车转向架故障特征提取 [J]. 西南交通大学学报, 2014, 49(1): 27-32.

QIN Na, JIN Weidong, HUANG Jin, et al. Feature extraction of high speed train bogie based on ensemble empirical mode decomposition and sample entropy[J]. Journal of Southwest Jiaotong University， 2014， 49(1): 27-32.

[9] 翟冰, 金炜东, 秦娜. 基于 EEMD 和球 SVM 的高速列 车转向架故障估计 [J]. 计算机测量与控制, 2014,22(8): 2356-2369.

ZHAI Bing, JIN Weidong, QIN Na. Running state estimation of high-speed train based on EEMD and hyper-sphere support vector machines[J]. Computer Measurement and Control, 2014, 22(8): 2356-2369.

[10] 宋神. 基于 CEEMD 和特征融合的高速列车振动信号 特征分析[D]. 成都: 西南交通大学, 2016 .

SONG Yi. Characteristic analysis of vibration signals of high speed train based on CEEMD and feature fusion[D]. Chengdu: Southwest Jiaotong University, 2016.

[11] 井波. 基于 EEMD 的高速列车安全性态数据的特征研 究[D]. 成都: 西南交通大学, 2015 .

JING Bo. Research on characteristics of safety status data of high speed train based on EEMD[D]. Chengdu: Southwest Jiaotong University, 2015.

[12] CHEN Q, HUANG N, RIEMENSCHNEIDER S, et al. A B-spline approach for empirical mode decompositions[J]. Advances in Computational Mathematics, 2006, 24(1): 171-195.

[13] 樊志平, 张歌凌. EMD 中包络算法改进的研究与分析 [J]. 计算机仿真, 2010, 27(6): 126-129.

FAN Zhiping, ZHANG Geling. The research of improved envelope algorithm of EMD[J]. Computer Simulation, 2010, 27(6): 126-129.

[14] KOPSINIS Y, MCLAUGHLIN S. Improved EMD using doubly-iterative sifting and high order spline interpolation[J]. Eurasip Journal on Advances in Signal Processing, 2008(1): 1-8.

[15] 朱伟芳, 赵鹤鸣, 陈小平. 一种最小长度约束的 EMD 包络拟合方法 [J]. 电子学报, 2012, 40(9): 1909-1912. ZHU Weifang, ZHAO Heming, CHEN Xiaoping. A least-length constrained envelope approach for EMD [J]. Acta Electronica Sinica, 2012, 40(9): 1909-1912.

[16] ZHAO J P, HUANG D J. Mirror extending and circular spline function for empirical mode decomposition method[J]. Journal of Zhejiang University Science A, 2001, 2(3): 247-252.

[17] DENG Y, WANG W, QIAN C, et al. Boundary processing technique in EMD method and Hilbert transform[J]. Chinese Science Bulletin, 2001，46(11): 954-961.

[18] RAVINDRA B, MALLIK A K. Performance of non-linear vibration isolators under harmonic excitation[J]. Journal of Sound and Vibration, 1994， 170(3): 325-337.

[19] WU Q, RIEMENSCHNEIDER S D. Boundary extension and stop criteria for empirical mode decomposition[J]. Advances in Adaptive Data Analysis, 2010,2(2): 157-169.

[20] RILLING G, FLANDRIN P, GONCALVES P. On empirical mode decomposition and its algorithms[C]// Proceedings of IEEE-EURASIP Workshop on Nonlinear Signal and Image Processing. Grado: IEEE, 2003: 8-11.

[21] LIN J, QU L. Feature extraction based on morlet wavelet and its application for mechanical fault diagnosis[J]. Journal of Sound and Vibration, 2000, 234(1): 135-148.

[22] LIU Z, ZUO M J, JIN Y, et al. Improved local mean decomposition for modulation information mining and its application to machinery fault diagnosis[J]. Journal of Sound and Vibration, 2017, 397: 226-281.

[23] WU Z H, HUANG N E. Ensemble empirical mode decomposition: A noise-assisted data analysis method[J]. Advances in Adaptive Data Analysis, 2011, 1(1): 1-51.

[24] EMD with a soft sifting stopping criterion[DB/OL]. https: //ww2.mathworks.cn/matlabcentral/fileexchange/70032-e md-with-soft-sifting-stopping-criterion.

[25] Case Western Reserve University. Bearing data center[DB/OL]. http: //csegroups.case.edu/bearing data center/pages/welcome-case-western-reserve-university-be aring-data-center-website.

[26] SMITH W A, RANDALL R B. Rolling element bearing diagnostics using the Case Western Reserve University data: A benchmark study[J]. Mechanical Systems and Signal Processing, 2015, 64-65: 100-131.

[27] FENG Z, ZUO M J. Vibration signal models for fault 
diagnosis of planetary gearboxes[J]. Journal of Sound and Vibration, 2012, 331(22): 4919-4939.

作者简介: 彭丹丹, 女, 1993 年出生。主要研究方向为振动信号处理、 旋转机械故障诊断。

E-mail: dandan_peng@qq.com

刘志亮(通信作者), 男, 1984 年出生, 博士, 副教授。主要研究方向为
智能传感与状态监测、信号处理与数据挖掘、寿命预测与故障诊断。 E-mail: zhiliang_liu@uestc.edu.cn

靳亚强, 男, 1991 年出生, 硕士研究生。主要研究方向为旋转机械故障 诊断。

E-mail: yaqiang.jin@qq.com

秦勇, 男, 1971 年出生, 博士，教授。主要研究方向为轨道交通安全保 障和运输组织、智能交通系统。

E-mail: yqin@bjtu.edu.cn 